\title{
Trabajo independiente interdisciplinar en programas académicos de contabilidad y finanzas
}

\author{
Interdisciplinary independent work in academic \\ accounting and finance programs
}

DOI: http://dx.doi.org/10.17981/cultedusoc.10.2.2019.09

Recibido: 11/03/2019. Aceptado: 16/08/2019

\author{
Libia Bermúdez Chaveco \\ Universidad de Holguín (Cuba) \\ lbermudezc@uho.edu.cu \\ Misleidis Teope Romero \\ Universidad de Holguín (Cuba) \\ lbermudezc@uho.edu.cu
Esnaidel Rodríguez Garcel
Universidad de Holguín (Cuba)
lbermudezc@uho.edu.cu

Para citar este artículo:

Bermúdez, L., Teope, M. y Rodríguez, E. (2019). Trabajo independiente interdisciplinar en programas académicos de contabilidad y finanzas. Cultura, Educación y Sociedad, 10(2). 109-122. DOI: http://dx.doi.org/10.17981/cultedusoc.10.2.2019.09

\section{Resumen}

La independencia cognitiva del estudiante implica procesos de resignificación pedagógica, centrados en el trabajo independiente y autónomo de naturaleza interdisciplinar, que se corresponda con el modelo de profesional concebido en la educación superior. Desde esta perspectiva la mediación didáctica centrada en la tarea del docente, resulta un proceso clave para la dirección del trabajo independiente. En este artículo, se caracterizan los componentes estructurales y dinámica funcional de una propuesta didáctica orientada a fortalecer el desarrollo del trabajo independiente interdisciplinario, de manera que se propicie el desarrollo humano y social de los estudiantes. El fundamento metodológico se organiza en atención a secuencias didácticas que atienden la lógica del proceso de construcción de conocimiento; por ello se cubren etapas de diagnóstico, formulación, orientación y evaluación de la actividad independiente interdisciplinar en el ámbito de la enseñanza, en programas académicos de Contabilidad y Finanzas. Entre los resultados destaca que, la selección de núcleos estructurantes por disciplina permite la formulación coherente de trabajo independiente interdisciplinar. Se concluye que la propuesta de secuencias didácticas garantiza una adecuada y flexible estructuración de los nexos disciplinarios, constituye una alternativa válida y pertinente para el trabajo de los colectivos pedagógicos que convergen en la construcción de los espacios cognitivos interdisciplinarios.

Palabras clave: Mediación didáctica; trabajo independiente; interdisciplinariedad

\section{Abstract}

The cognitive independence of the student implies processes of pedagogical resignification, focused on independent and autonomous work of an interdisciplinary nature, corresponding to the professional model conceived in higher education. From this perspective, didactic mediation focused on the teacher's task is a key process for the direction of independent work. In this article, the structural components and functional dynamics of a didactic proposal aimed at strengthening the development of interdisciplinary independent work are characterized, so as to promote the human and social development of students. The methodological foundation is organized in attention to didactic sequences that address the logic of the process of knowledge construction; Therefore, diagnostic, formulation, orientation and evaluation stages of interdisciplinary independent activity in the field of teaching in academic Accounting and Finance programs are covered. Among the results it is worth highlighting that, the selection of structuring nuclei by discipline allows the coherent formulation of interdisciplinary independent work. It is concluded that the proposal of didactic sequences guarantees an adequate and flexible structuring of disciplinary links, constitutes a valid and pertinent alternative for the work of pedagogical groups that converge in the construction of interdisciplinary cognitive spaces.

Keywords: didactic mediation; independent work; interdisciplinary 


\section{INTRODUCCIÓN}

Las exigencias que la sociedad le ha planteado a la Educación Superior están sustentadas en las transformaciones que se operan desde lo social, económico y ambiental, afianzando la pertinencia de la misma. Es precisamente en este escenario donde la universalización de la educación superior reviste significación especial, pues desde su concepción, asume como rasgo esencial en el proceso de enseñanza-aprendizaje al estudiante como centro de la actividad, para que este sea capaz de asumir de modo activo su propio proceso de formación.

El proceso enseñanza- aprendizaje es el escenario donde el estudiante con diferentes niveles de complejidad, identifica y busca la posible solución a problemas de su esfera de actuación profesional, las cuales pueden ser modeladas a través de situaciones pedagógicas desde lo académico, investigativo y laboral. Es en este ámbito donde la interdisciplinariedad juega un papel estimulante y desarrollador para el estudiante universitario.

Específicamente en la educación superior, es necesario estimular que el futuro profesional consagre de manera productiva y creativa el tiempo dedicado a la realización del trabajo independiente, a partir de la concepción del modelo de formación del profesional explícito en la norma. En el caso de Programas Académicos de Contabilidad y Finanzas los perfiles apuntan a personas altamente calificadas para resolver los problemas que presentan las ciencias contables y financieras en el mundo actual.

A través de la construcción argumentativa se abordan como principales categorías de análisis el trabajo independiente y la interdisciplinariedad. Autores como Pidkasisti (1986), Rojas (1978), Álvarez (1998), Díaz (1998), Ortiz y Mariño (2003) y Addine
(2004) coinciden en señalar que el trabajo independiente puede considerarse un método, un medio, una vía, un sistema, una forma de aprendizaje, un medio de organización de la actividad y un proceso.

Con relación a la interdisciplinariedad, investigadores como Álvarez (1988) aborda las características que debe tener una tarea docente interdisciplinaria: dominio de los contenidos de las diferentes disciplinas por parte de los docentes, trabajo cooperado y sistemático entre docentes de un mismo grado o año y conocer las características que debe tener una tarea docente. Por otro lado, Martínez y Addine (2011) señalan que el problema docente interdisciplinario presupone el planteamiento o identificación de contradicciones en el objeto de la profesión, llevadas a situaciones de enseñanza-aprendizaje para motivar la búsqueda y estimular el establecimiento de nexos y de relaciones interdisciplinarias para su solución. Coincide Marín (2010) cuando explica la construcción de un conocimiento interdisciplinario con base a flujos de relación que provienen de los dominios de las diferentes disciplinas Desde las ciencias económicas autores como Settimi, Quartucci, Audino (s.f.) suponen la concreción de un diálogo provechoso entre disciplinas científicas que tienen sus propias especificidades.

Lo analizado por Ivarola (s.f.) destaca la idea de que la interdisciplinariedad puede llegar a mejorar tanto el carácter explicativo como predictivo de una teoría económica, pero aborda algunas limitaciones que hay que tener en cuenta como la condición necesaria que las disciplinas integradas al trabajo conjunto con la economía hayan validado teorías, reconociendo el problema de la multiplicidad de factores relevantes, que dificultan tanto la predicción como la explicación, no solo en lo conciernente a la economía, sino a las ciencias sociales en general. 
Se asume lo abordado por Martínez y Addine (2014) sobre la utilización del problema profesional como eje integrador del proceso de enseñanza-aprendizaje interdisciplinario requiere para su instrumentación en la práctica de un determinado arreglo didáctico. Este se logra a través del establecimiento de una relación entre el problema profesional, los problemas docentes interdisciplinarios y las tareas docentes interdisciplinarias en su ejercicio integrador.

La diversidad de criterios acerca de cómo influir desde el proceso de enseñanzaaprendizaje en la formulación del trabajo independiente con marcado carácter interdisciplinar y el logro de modos de actuación que se caractericen por la independencia y creatividad en la solución de problemas profesionales, es motivo de obligada reflexión para todos los que se encuentran inmersos en la actividad docente. Al respecto surge el aprendizaje como una categoría fundamental, por cuanto desde los aportes de Cárdenas y Ángulo (2016), el aprendizaje implica procesos de adaptación al contexto que conllevan diferentes formas de actuación, una de ellas podría estar asociada al trabajo interdisciplinar, cuyo Es por ello que el trabajo tiene como objetivo la elaboración de acciones didácticas que potencien el trabajo independiente con enfoque interdisciplinar desde las disciplinas Teoría Económica y Contabilidad en la carrera de Contabilidad y Finanzas.

Fundamento METOdológico DE LA PROPUESTA DE TRABAJO INTERDISCIPLINARIO

\section{Etapas por las que transitan las acciones didácticas y los modelos elaborados}

El problema docente interdisciplinario según demuestran Martínez y Addine (2014) presupone el planteamiento o identificación de contradicciones que se manifiestan en el objeto de la profesión, que llevadas a situa- ciones de enseñanza-aprendizaje motivan la búsqueda y estimulan el establecimiento de nexos y de relaciones interdisciplinarias para su solución.

Siendo consecuente con lo expuesto por reconocidos investigadores y las discusiones profesionales devenidas de las experiencias en la práctica profesional en la educación superior se revela que, las acciones didácticas interdisciplinarias son aquellas que dinamizan la gestión de dirección del proceso de enseñanza aprendizaje a corto plazo con un enfoque problémico y profesionalizador.

En este sentido la propuesta fundamentada en secuencias didácticas transitan por cuatro etapas: diagnóstico, formulación, orientación y evaluación.

Diagnóstico: Se diagnostican las potencialidades que brinda la interdisciplinariedad en las disciplinas Teoría económica y Contabilidad para reforzar el trabajo independiente.

Formulación: Se caracterizan las potencialidades que brinda la interdisciplinariedad en las disciplinas Teoría Económica y Contabilidad para potenciar el trabajo independiente en la modalidad CPE.

Orientación: Asegura una adecuada comunicación de la tarea docente para su comprensión por los estudiantes en la realización del trabajo independiente interdisciplinar en las disciplinas Teoría Económica y Contabilidad.

Evaluación: Se valora el comportamiento del trabajo independiente interdisciplinar en la disciplina Teoría Económica y se actualiza el diagnóstico.

La estructura didáctica de los modelos que materializan a las acciones se enmarca en primer lugar al objetivo como elemento orientador del proceso, representa la modelación subjetiva del resultado esperado y está condicionado por las exigencias sociales de una determinada época (Addine, 2004). 
Se reconoce al contenido como la parte de la cultura seleccionada con sentido pedagógico para la formación integral del educando, lleva implícito las potencialidades para que el hombre lo enriquezca (Álvarez, 1995).

Se admite que el método constituye el sistema de acciones que regulan la actividad del profesor y los alumnos, en función del logro de los objetivos, atendiendo a los intereses y motivaciones de estos últimos y a sus características particulares (Silvestre y Zilbersteins, 2000)

Se considera que los procedimientos son detalles del método, es una operación particular práctica o intelectual de la actividad del profesor o de los alumnos complementa la forma de asimilación de los conocimientos que presupone determinado método (Labarrere y Valdivia, 1989)

Dentro de las formas de organización del proceso enseñanza aprendizaje a pesar de reconocer que en todas pueden implementarse las acciones propuesta; fue seleccionada las clases en modalidad Curso por Encuentro por ser la que con mayor frecuencia se trabaja en la enseñanza semipresencial. Tiene como objetivos aclarar las dudas correspondientes a los contenidos y actividades previamente estudiados por los alumnos, debatir $\mathrm{y}$ ejercitar dichos contenidos y evaluar su cumplimiento, así como explicar los aspectos esenciales del nuevo contenido y orientar el trabajo independiente correspondiente.

Por último, se tuvo en cuenta a la evaluación del aprendizaje, en su carácter integrador, holístico y personológico, como proceso y resultado. Es el componente que influye en todo el proceso de desarrollo de la personalidad del estudiante, potencia la independencia, la metacognición, las relaciones interpersonales, la reflexión, la responsabilidad y la práctica creativa. Permite valorar cualitativa y cuantitativamente los cambios que se producen en el aprendizaje. Genera un crecimiento individual y grupal (Mena, 2001).

Las relaciones interdisciplinarias en la concepción y práctica curricular de programas académicos de Contabilidad y Finanzas. Contexto de intervención: Centro Universitario Municipal de Banes, Cuba

El Centro Universitario Municipal de Banes, Cuba, graduó en sus quince años de fundada 298 profesionales de las ciencias contables y financieras. Tras el proceso de integración de la educación superior y el inicio de la modalidad del Curso por Encuentro se dio reapertura a la carrera de Licenciatura en Contabilidad y Finanzas, con una matrícula actual de 78 estudiantes de 1er a 4to año, en tres planes de estudio: Plan D, Plan D modificado y Plan E. su objetivo general es formar profesionales para resolver problemas relacionados con los procesos contables y financieros. Es importante que el abordaje del objeto de la formación se de en correspondencia con el desarrollo científico actual de estas ramas del saber, de forma creativa. Se propende a la independencia cognitiva, aplicación de normas y principios de la ética y la estética de los profesionales de la contabilidad y las finanzas con un alto sentido de responsabilidad y compromiso político y social.

La disciplina Teoría Económica se ubica en la cuarta posición dentro del plan de estudio, antecedida por disciplinas como: Matemática, Contabilidad y Administración. Integra las asignaturas Economía Política I, se imparte en el 1er semestre de la carrera; Economía Política II, en el 2 semestre; Economía Política III, en el 3 semestre; Pensamiento Económico Universal, 4 semestre y Pensamiento Económico Cubano y Economía cubana en el 5to semestre, todas con 32 horas clases, representando cada una el $32 \%$ de representatividad. 


\section{Relación con otras disciplinas}

Desde la disciplina Teoría Económica se aporta a las demás disciplinas de manera general el desarrollo de la convicción ética que supone las funciones que realiza este profesional, bajo los principios de la honestidad científica, como la capacidad de enfrentarse a los problemas socioeconómicos en defensa de los intereses de la sociedad y el ejercicio de la crítica y la autocrítica oportuna en la esfera de actuación. Esto se revierte en especial atención en disciplina como Contabilidad cuando se cumplen con los procedimientos, normas y principios que rigen la misma, contribuyendo a análisis exhaustivos que contrarrestan la ocurrencia de hechos delictivos; en la disciplina de Finanzas en todo el proceso financiero, en la de Costo pues contribuye al análisis de la causaefecto y por ende una acertada toma de decisiones.

A pesar de la representatividad de la disciplina y su aporte al plan de estudio no siempre se logra que el estudiante reconozca la importancia de la misma para su formación y desempeño profesional, lo que conlleva a desmotivación por la misma.

Como característica peculiar, de la modalidad semipresencial Curso por Encuentro, está precisamente en que el estudiante asistido por el profesor autogestiona el conocimiento a través de la bibliografía básica y complementaria, con el empleo de las tecnologías de la información y las comunicaciones, y las potencialidades que ofrece el entorno.

El diagnóstico se sustentó en la búsqueda del conocimiento en profesores, estudiantes y empleadores sobre el trabajo independiente con enfoque interdisciplinar dentro del proceso enseñanza aprendizaje. La constatación inicial permitió una reconstrucción reflexiva sobre la gestión del conocimiento en la temática y a partir de la matriz de marco lógico se determinaron las fortalezas y debilidades en la dirección del proceso de enseñanzaaprendizaje relacionadas con el trabajo independiente interdisciplinar en la disciplina Teoría Económica.

La triangulación de los resultados derivados de la revisión documental a expedientes de las asignaturas, las visitas a clases y las evaluaciones aplicadas, evidenció inconsistencia en la selección y planificación de los nexos disciplinarios a emplear, por lo que desde el objetivo no se intenciona el o los nexos (s) interdisciplinarios a emplear y no favorece los vínculos con los resultados que como experiencia poseen los estudiantes de su práctica pre profesional.

Un representativo número de estudiantes no reconocen los resultados económicos contables emanados de las formas de gestión estatal y no estatal territorial como fuentes bibliográficas de inagotable valor para la disciplina Teoría Económica, esto evidencia carencias de actividades articuladas entre disciplinas desde el plan metodológico.

\section{Diseño de las secuencias didácticas que potencien el trabajo independiente con relaciones interdisciplinarias}

A continuación, se exponen las acciones didácticas que potencian al trabajo independiente con enfoque interdisciplinar. Desde la disciplina Teoría Económica, se asocia la Contabilidad como principal dominio científico para establecer los vínculos disciplinarios. 


\section{Acciones para el diagnóstico de las potencialidades para reforzar el trabajo independiente desde la interdisciplinariedad en las disciplinas Teoría económica y Contabilidad en la modalidad Curso por Encuentro.}

Objetivo: Diagnosticar las potencialidades que brinda la interdisciplinariedad en las disciplinas Teoría económica y Contabilidad para reforzar el trabajo independiente.

Fundamentación: Estas acciones están dirigidas en lo fundamental a diagnosticar cómo se manifiestan y emplean las potencialidades que brinda la interdisciplinariedad en las disciplinas Teoría Económica y Contabilidad para fortalecer el trabajo independiente. Es el docente quien realiza el mismo utilizando como escenario el colectivo de las disciplinas, el aula y el intercambio con las entidades territoriales donde están vinculados los estudiantes.

Se aplica el método de la observación, se sintetizan las regularidades que son de interés para el colectivo de la disciplina, clasificándolos a partir de los tres grupos que intervienen: profesores, estudiantes y entidades territoriales, como procedimiento se utiliza la formulación de un cuestionario para la observación y el trabajo en equipo. Los medios seleccionados parten del expediente de las asignaturas que comprende cada disciplina para la determinación de potencialidades, nexos interdisciplinarios, objetivos de cada año, estrategias curriculares, bibliografía entre otras.

Se intercambian experiencias pedagógicas que se derivan de las prácticas en el desarrollo de la clase encuentro como forma de organización seleccionada. El muestreo a la evaluación sistemática, parcial y final contribuye a revelar el enfoque de las actividades concebidas con una perspectiva disciplinar o interdisciplinar.
Todo ello presupone:

1. En los docentes: Es el eslabón primario si se reconoce al profesor como dirigente de dicho proceso.

a. Diagnóstico de las prácticas pedagógicas que se emplean:

- Fundamentación de las disciplinas Teoría Económica y Contabilidad.

- Se determinan las competencias profesionales refrendadas en los objetivos de cada año.

- Se determinan los intereses y móviles de aprendizaje.

- Se tienen en cuenta las experiencias devenidas de la práctica profesional para impulsar nuevas metas.

- Determinación de los nodos interdisciplinarios para la formulación del mismo.

2. En los estudiantes presupone:

b. Diagnóstico de las prácticas de aprendizaje que se emplean:

- Revelan expectativas profesionales.

- Aprecian la relevancia de su práctica profesional y el sentido de nuevas metas.

- Estado de aceptación de las disciplinas del currículo de los tres primeros años de la carrera.

3. Para las entidades territoriales presupone:

c. Diagnóstico de las habilidades profesionales que se producen de acuerdo con el tipo de actividad.

- Expresan intereses en el nivel de desempeño. 
- Actualizan el banco de problemas y resultados obtenidos derivados de modificaciones estructurales y funcionales amparados por la legislación vigente.

- Experiencias derivadas del ejercicio de la profesión en los graduados de la especialidad de los últimos cinco años.

\section{Acciones para potenciar la formulación del trabajo independiente desde la interdisciplinariedad en las disciplinas Teoría económica y Contabilidad.}

Objetivo: Caracterizar las potencialidades que brinda la interdisciplinariedad en las disciplinas Teoría Económica y Contabilidad para potenciar el trabajo independiente en la modalidad Curso por Encuentro.

Fundamentación: Las acciones están dirigidas en lo fundamental a caracterizar las potencialidades que brinda la interdisciplinariedad en las disciplinas Teoría Económica y Contabilidad para reforzar el trabajo independiente. Es el docente quien realiza el mismo utilizando como escenario el colectivo de las disciplinas, el aula y el intercambio con las entidades territoriales donde están vinculados los estudiantes.

Se tienen en cuenta las acciones para el desarrollo de competencias profesionales, dentro de las que se encuentran habilidades como:

- Caracterizar: Analizar las peculiaridades del objeto y determinar los elementos más relevantes.

- Comparar: analizar las peculiaridades del objeto, determinar semejanzas y diferencias a partir de los indicadores dados, se ejemplifica.
- Explicar: establecer las causas y condiciones que determinan la ocurrencia de determinado objeto o fenómeno (relación causa-efecto), comparar con otros objetos y fenómenos, determinar las características esenciales del objeto o fenómeno, definir el concepto y ejemplificar las manifestaciones concretas del objeto o fenómeno.

- Valorar. Caracterizar el objeto de valoración, establecer los criterios de valoración, comparar el objeto con los criterios de valor establecidos, elaborar juicios de valor acerca del objeto de acuerdo con sus características y los criterios de valor establecidos.

La aplicación de métodos orales, dentro de ellos el de explicación, favorece que los docentes muestren la lógica del estudio realizado; como procedimiento se utiliza el trabajo con el texto pues se determina lo esencial, se realizan cuadros que aseguren la fluidez entre los nexos disciplinarios. Dentro de los vínculos disciplinarios seleccionados se encuentran los observados en la Tabla 1.

Los medios que se emplean son: el programa de las asignaturas seleccionadas de ambas disciplinas, la bibliografía básica y complementaria, el plan de clases, el sistema de actividades que aparece en la plataforma interactiva MOODLE, la bibliografía, los resultados económicos contables de las principales entidades del territorio entre otras.

Es posible escoger el momento de la clase encuentro en que es más factible el desarrollo del mismo. La frecuencia y cantidad de tareas que cumplan con la intencionalidad interdisciplinar está en correspondencia con las potencialidades que brindan los contenidos, debe ser fluido y coherente. 
TABLA 1

Nexos disciplinarios.

\begin{tabular}{ll}
\hline \multicolumn{1}{c}{ Disciplina Teoría Económica } & \multicolumn{1}{c}{ Disciplina Contabilidad } \\
\hline $\begin{array}{l}\text { Las relaciones monetario-mercantiles en la } \\
\text { construcción del socialismo en Cuba. }\end{array}$ & $\begin{array}{l}\text { Partidas fundamentales que integran el Activo Circulante, } \\
\text { como son el Efectivo, tanto en Caja como en Banco, la } \\
\text { problemática relacionada con cuentas por cobrar. }\end{array}$ \\
$\begin{array}{l}\text { Sistema Presupuestario de Financiamiento } \\
\text { y el Cálculo Económico en la dirección de la } \\
\text { economía socialista }\end{array}$ & Normas y principios de la Contabilidad \\
$\begin{array}{l}\text { Equilibrio entre el plan y el mercado en la } \\
\text { construcción del socialismo. }\end{array}$ & $\begin{array}{l}\text { Registrar y valorar correctamente diferentes tipos de } \\
\text { inventarios, como mercancías, útiles y herramientas y } \\
\text { envases. }\end{array}$ \\
$\begin{array}{l}\text { Transformaciones económicas relacionadas con } \\
\text { el manejo y control de los recursos materiales y } \\
\text { financieros. }\end{array}$ & $\begin{array}{l}\text { Cuentas del activo y pasivo circulante } \\
\text { Sistema de Administración Fiscal. }\end{array}$ \\
$\begin{array}{l}\text { Registrar operaciones vinculadas con los impuestos y } \\
\text { contribuciones. }\end{array}$ \\
$\begin{array}{l}\text { Registrar las operaciones vinculadas con el pasivo } \\
\text { circulante: letra de cambio. }\end{array}$ \\
$\begin{array}{l}\text { Taptión de los recursos financieros. } \\
\text { cubana. }\end{array}$ & $\begin{array}{l}\text { Aplicas técnicas de valuación, registro y presentación de } \\
\text { Balance General }\end{array}$ \\
\hline
\end{tabular}

Fuente: Programa de las Disciplinas.

Se planifica la medición de los resultados en la evaluación sistemática, parcial o final a emplear.

Se seleccionan entidades del territorio para el análisis de hechos contables con repercusión para sus vínculos con la teoría económica estudiada: una empresa municipal y tres Unidades Empresariales de Base.

Se resume:

1. Para los docentes presupone:

- Selección de los núcleos de cada disciplina que permitan coherentemente la formulación de actividades de trabajo independiente interdisciplinar.

- Frecuencia con que se plantean actividades de trabajo independiente con enfoque interdisciplinar desde las disciplinas Teoría Económica y Contabilidad.
- Competencias profesionales que plantean desarrollar las disciplinas Teoría Económica y Contabilidad y priorizan la concepción del trabajo independiente.

2. Para los estudiantes presupone:

- Nociones que recibe sobre los vínculos disciplinares en aras de modelar las competencias profesionales refrendadas en el Modelo del Profesional de la especialidad.

- Niveles de realización, satisfacción y preparación en las actividades de trabajo independiente con enfoque interdisciplinar.

- Competencias profesionales que se desarrollan a través del trabajo independiente con relaciones interdisciplinarias entre las disciplinas Teoría Económica y Contabilidad. 
3. Para las entidades estatales presupone:

- Utilización de las experiencias prácticas y/o investigativas de los profesores, tutores y egresados vinculados a las esferas productivas, de los servicios y formas no estatales de empleo.

- Actividades de trabajo independiente que en su realización participen especialistas de las entidades y la Asociación Nacional de Economistas de Cuba.

- Las actividades que se prevén vinculan al estudiante con su entorno en las esferas de la producción, los servicios o formas no estatales de empleo.

\section{Acciones para potenciar la orientación del trabajo independiente en las disciplinas Teoría Económica y Contabilidad.}

Objetivo: Comunicar la tarea docente que asegure la comprensión para la realización del trabajo independiente interdisciplinar en las disciplinas Teoría Económica y Contabilidad.

Fundamentación: La acción está dirigida en lo fundamental a asegurar una adecuada comunicación de la tarea docente para potenciar el trabajo independiente interdisciplinar en la disciplina Teoría Económica con vínculos disciplinarios con Contabilidad. Es el docente quien realiza el mismo utilizando como escenario el grupo de estudiantes y otros actores externos del proceso enseñanza aprendizaje como las entidades estatales, Asociación Nacional de Economistas y Contadores de Cuba.

Se emplea el método de trabajo independiente, es uno de los más empleados en la educación superior pues el estudiante realiza tareas de manera independiente bajo la dirección del profesor. Como procedimientos se utilizan el explicativo ilustrativo pues asegura la comprensión del mismo, la consulta en fuentes bibliográficas (en soporte de papel y digital)

Los medios que se emplean son la bibliografía básica y complementaria, el sistema de actividades que aparece en la plataforma interactiva MOODLE, los resultados económicos contables de las entidades del territorio seleccionadas.

Se realiza en el momento de la clase encuentro en que se considera más factible para el desarrollo del mismo (al inicio, en el medio o al final de la clase).

Se precisa cómo se medirán los resultados a través de la evaluación sistemática.

Ello presupone:

1. En los docentes:

b. Se aprecia que la orientación garantiza la solución del problema profesional planteado y un aseguramiento de los resultados (para qué, qué, a quién y cómo)

c. Se realiza de manera clara, precisa y fluida de acuerdo con las características de los estudiantes.

d. Se establece empatía con los estudiantes.

1. Para los estudiantes presupone:

b. Formas en que manifiesta la comprensión del trabajo independiente interdisciplinar a través de:

- Variedad de fuentes bibliográficas actualizadas a las que se accede. Empleo de las TIC

- Si realizan análisis y síntesis

- Si revelan determinar lo esencial.

- Si establecen vínculos causales. 
IV. Acciones para potenciar la evaluación del trabajo independiente interdisciplinar en las disciplinas Teoría Económica y Contabilidad

Objetivo: Valorar el comportamiento del trabajo independiente interdisciplinar en la disciplina Teoría Económica

Fundamentación: Las acciones están dirigidas en lo fundamental a valorar el comportamiento del trabajo independiente interdisciplinar en la disciplina Teoría Económica.

Se mantiene el empleo del método de trabajo independiente de forma secuencial. Como procedimientos se utilizan la elaboración de nuevos ejemplos, visita a entidades laborales del territorio entre otros. Los medios que se emplean son la bibliografía básica y complementaria, el sistema de actividades que aparece en la plataforma interactiva MOODLE, los resultados económicos contables de las entidades del territorio seleccionadas, el pizarrón entre otros.

Se realiza en el primer momento de la clase encuentro dirigido a la aclaración de dudas y a la evaluación del mismo.

Se emplea la autoevaluación, la coevaluación y la evaluación. Se actualiza el diagnóstico existente.

Es el colectivo pedagógico de la disciplina, estudiantes seleccionados y miembros de las entidades estatales quienes realizan el debate profesional final, utilizando como escenario el taller científico metodológico. Se actualiza el diagnóstico y se proyectan acciones con los grupos científicos estudiantiles.

Lo mismo se resume en:

a. Dominio de las exigencias del currículo para su desarrollo

b. Si se contribuye a la materialización de las aspiraciones planteadas en el perfil profesional.

c. Se cuenta con la participación social para la solución de la problemática de la profesión en el entorno d. Se evidencia una vinculación de la teoría, la práctica y la investigación.

e. Se utilizan fuentes bibliográficas actualizadas y se emplean las TIC.

f. Resultados que se obtienen cuantitativos y cualitativos en la realización del trabajo independiente por los estudiantes en la disciplina Teoría Económica. (Actualización del diagnóstico)

g. Propuesta de nuevas acciones que involucren a los grupos científicos estudiantiles.

\section{INSTRUMENTACIÓN DE LAS ACCIONES}

Los ejemplos que se proponen se concibieron a partir de la disciplina Teoría Económica con vínculos disciplinarios con Contabilidad, siguiendo la lógica de las acciones formuladas, los componentes del proceso de enseñanza-aprendizaje y los comentarios metodológicos:

\section{Ejemplo 1}

Objetivo: Explicar cómo se manifiestan las relaciones monetario-mercantiles en la construcción del socialismo en Cuba, lo que favorece la comprensión de la viabilidad del proyecto socialista y la actualización del modelo económico cubano.

Contenido: Las relaciones monetario-mercantiles en la construcción del socialismo en Cuba.

Método: Trabajo independiente.

Medios de Enseñanzas:

Bibliografía de consulta para estudiantes:

1. Colectivo de Autores, Economía Política de la Construcción del Socialismo: Fundamentos Generales, Editorial Félix Varela, La Habana. Págs.148 - 153

2. Frías, R. (Ed). Las relaciones monetariomercantiles en la construcción del socialismo. La Habana: Editorial Félix Varela. 
3. Maldonado. Estudio de la Contabilidad General

4. Horngreen. Contabilidad.

5. Lineamientos de la Política Económica y Social del Partido y la Revolución.

6. UBE Pecuario. Estado de Resultado (N. 2). Recuperado de Dirección Municipal de Economía y Planificación. Banes. Holguín.

Forma de Organización: Clase encuentro Evaluación: Oral y escrita

Comentario metodológico: La tarea docente se concibe desde la asignatura Economía Política III, empleando los núcleos disciplinarios de la asignatura Contabilidad General II (partidas fundamentales que integran el Activo Circulante, como son el Efectivo, tanto en Caja como en Banco, la problemática relacionada con cuentas por cobrar). Está llamado a desarrollar la capacidad crítica respecto a las relaciones monetario-mercantiles en la construcción del socialismo en Cuba lo que favorece la comprensión de la viabilidad del proyecto socialista y la actualización del modelo económico cubano, empleando la bibliografía antes referenciada. Se parte del análisis de un asiento contable de una entidad del entorno local. La unidad entre teoría y práctica potencia el desarrollo del trabajo independiente al insistir constantemente en el método de la Economía Política, a fin de asegurar que prevalezca el análisis de los fenómenos y su enfoque partiendo de los intereses sociales.

Tarea Docente: La Unidad Básica de Producción $\mathrm{X}$ de la localidad tiene dentro de su encargo social aprobado la producción de carne bobino. Al cierre del primer trimestre del año para lograr el cumplimiento de su plan al $100 \%$ y el $25 \%$ con respecto al año, contrata con la UEB Pecuaria la siguiente venta:

- Con fecha x vende $1000 \mathrm{~kg}$ de carne cuyo precio es de $0.80 \mathrm{ctv}$ el $\mathrm{kg}$.

- Con fecha x (veinte días posteriores a su emisión) se recibe el pago de la factura de dicha venta.

Explica cómo se manifiestan las relaciones monetario-mercantiles a partir de los hechos económicos contabilizados en el Estado de Resultado de la UEB Pecuaria (Tabla 2).

TABla 2.

Asiento contable.

\begin{tabular}{llll}
\hline Descripción & Parcial & Debe & Haber \\
\hline Cuentas por cobrar & & xxxxx & \\
UEB Pecuaria & xxxxx & & \\
Venta & & & $\operatorname{xxxx}$ \\
Contabilizando cuentas por cobrar por venta realizada. & & \\
Efectivo en Banco & & xxxxxx & \\
Costo de producción & & xxxxxx & \\
Cuenta por cobrar & & \\
UEB Pecuaria & xxxxxxx & & \\
Producción terminada & & & \\
Efectuando el cobro y fijando el costo. & & & \\
\hline
\end{tabular}

Fuente: Elaboración propia. 


\section{Ejemplo 2}

Objetivo: Explicar el papel de la valuación de los Activos Fijos Tangibles (AFT) en el financiamiento de la empresa cubana en el contexto de actualización del modelo económico cubano.

Contenido: papel de la valuación de los Activos Fijos Tangibles en el financiamiento de la empresa cubana

Método: Trabajo independiente.

Medios de Enseñanzas:

1. Colectivo de Autores. Economía Política de la Construcción del Socialismo: Fundamentos Generales, Editorial Félix Varela, La Habana.

2. Maldonado, R. Estudio de la Contabilidad General.

3. Horngreen, C. Contabilidad.

4. Lineamientos de la Política Económica y Social del Partido y la Revolución.

\section{Evaluación: Oral y escrita}

Comentario metodológico: La tarea docente se concibe empleando los núcleos disciplinarios de la asignatura Contabilidad General III (Aplicar técnicas de valuación, registro y presentación de las partidas fundamentales que integran el activo fijo del Balance General.) Su enfoque va dirigido a explicar el papel de la valuación de los
Activos Fijos Tangibles en el financiamiento de la empresa cubana en el contexto de actualización del modelo económico cubano, se contextualiza a partir de lo refrendado en los lineamientos de la política económica y social. Se parte de análisis realizado por una UEB productora del territorio cuya subordinación es nacional. El asiento contable da lugar al análisis.

Tarea Docente: La UEB Confitera y Molinera procede a realizar un avalúo de sus activos fijos tangibles contando con los datos observados en la Tabla 3.

Después de realizar el asiento contable correspondiente, explica qué papel le concede a la valuación (positivo o negativo) de los Activos Fijos Tangibles en el financiamiento de la misma, en el contexto de actualización del modelo económico cubano.

\section{Conclusiones}

Los elementos y exigencias que se tienen en cuenta para la propuesta de las acciones didácticas que contribuyen a potenciar el trabajo independiente interdisciplinar a partir de las disciplinas Teoría Económica y Contabilidad, garantizan una adecuada y flexible estructuración de los nexos disciplinarios, lo que constituye una herramienta de trabajo para el colectivo pedagógico de la disciplina.

TABla 3.

Asiento contable

\begin{tabular}{lll}
\hline Datos & Antes del Avalúo & Después del Avalúo \\
\hline Valor inicial en libro & $\$ 5000.00$ & $\$ 5800.00$ \\
Depreciación acumulada & 3000.00 & 3000.00 \\
Valor actual según libro & 2000.00 & 2800.00 \\
Incremento del valor & 800.00 & \\
\hline
\end{tabular}

Fuente: UEB Confitera y Molinera. 
La factibilidad de aplicación de las acciones didácticas para potenciar el trabajo independiente interdisciplinar posibilita corroborar su validez en el marco del proceso de enseñanza-aprendizaje en la formación del profesional de las ciencias contables y financieras.

Las tareas docentes de base interdisciplinar son concebidas en el ámbito de la mediación didáctica como estrategias de significativa pertinencia para fortalecer el currículo y contribuir a la formación integral de los profesionales en programas académicos de Contabilidad y Finanzas.

\section{REFERENCIAS}

Addine, F. (2004). Didáctica: Teoría y Práctica. La Habana: Pueblo y Educación.

Álvarez, C, M. (1998). La Pedagogía Universitaria. Una Expresión cubana. La Habana: Palacio de las Convecciones.

Cárdenas, N. y Angulo, F. (2016). Análisis de las dimensiones de adaptación, mejoramiento e innovación en los procesos de aprendizaje tecnológico. Cultura Educación y Sociedad, 7(2), 139-149. Recuperado de https://revistascientificas.cuc.edu.co/ culturaeducacionysociedad/article/ view/1468

Díaz, A. (1988). Didáctica y currículum. Convergencias en los programas de estudio. México D.F.: Nuevomar.

Ivarola, L. (s.f.). La economía desde una visión interdisciplinaria. En, $X V I$ Jornadas de Epistemología de las Ciencias Económicas. (pp. 146-162). Buenos Aires: CIECE.
Labarrere, G. y Valdivia, G. (1989). Pedagogía. La Habana: Pueblo y Educación.

Marín, F. (2010). Construcción de conocimiento sobre desarrollo sostenible desde una perspectiva inter y transdisciplinaria. En, Investigación en Ciencias Humanas. Estudios Posdoctorales. Colección Ciencias Humanas. Vol. I. (pp. 49-68). Universidad del Zulia, Venezuela.

Martínez, B. y Addine, A. (2014). Metodología para el diseño del proceso de enseñanza-aprendizaje interdisciplinario en la disciplina formación laboral investigativa. Opuntia Brava, 6(3), 1-11.

Martínez, B. y Addine, A. (2011). Metodología para el diseño del proceso de enseñanza-aprendizaje interdisciplinario en el nivel año, desde el eje integrador problema profesional. Opuntia Brava, 3(1). Recuperado de http://www.opuntiabrava.rimed.cu

Mena, E. (2001). Autoevaluación y creatividad: un reto para la Pedagogía Contemporánea. [Tesis Máster]. ISPEJV, La Habana.

Ortiz, E. y Mariño M. (2003). Problemas Contemporáneos de la Didáctica de la Educación Superior. CECECS. Universidad de Holguín.

Pidkasisti, P. I. (1986). La Actividad Cognoscitiva Independiente de los Alumnos en la Enseñanza. Ciudad de la Habana: Pueblo y Educación

Silvestre, M. y Zilbersteins, J. (2000). Aprendizaje y Diagnóstico. ¿Cómo hacer más eficientes el aprendizaje? México, D.F.: CEIDE. 
Libia Bermúdez Chaveco. Universidad de Holguín (Cuba).

Misleidis Teope Romero. Universidad de Holguín (Cuba).

Esnaidel Rodríguez Garcel. Universidad de Holguín (Cuba). 